\title{
Standardization of definitions in focal therapy of prostate cancer: report from a Delphi consensus project
}

\author{
A. W. Postema ${ }^{1}$ - T. M. De Reijke ${ }^{1} \cdot$ O. Ukimura ${ }^{3,4} \cdot$ W. Van den Bos ${ }^{1} \cdot$ \\ A. R. Azzouzi ${ }^{5}$ E. Barret ${ }^{6}$ - D. Baumunk ${ }^{7}$ - A. Blana ${ }^{8}$ - A. Bossi ${ }^{9}$ - M. Brausi ${ }^{10}$. \\ J. A. Coleman ${ }^{11} \cdot$ S. Crouzet ${ }^{12} \cdot$ J. Dominguez-Escrig ${ }^{13} \cdot$ S. Eggener ${ }^{14} \cdot$ R. Ganzer $^{15}$. \\ S. Ghai ${ }^{16}$ - I. S. Gill ${ }^{3}$ - R. T. Gupta ${ }^{17}$ - T. O. Henkel ${ }^{19} \cdot$ M. Hohenfellner ${ }^{20}$. \\ J. S. Jones ${ }^{21}$ - F. Kahmann ${ }^{19}$ - C. Kastner ${ }^{22}$ - K. U. Köhrmannn' 23 - G. Kovacs ${ }^{24}$. \\ R. Miano ${ }^{25}$ - R. J. van Moorselaar ${ }^{26} \cdot$ N. Mottet $^{27} \cdot$ L. Osorio ${ }^{28}$ - B. R. Pieters ${ }^{2}$. \\ T. J. Polascik ${ }^{18}$ - A. R. Rastinehad ${ }^{29}$ - G. Salomon ${ }^{30}$ - R. Sanchez-Salas ${ }^{6}$.

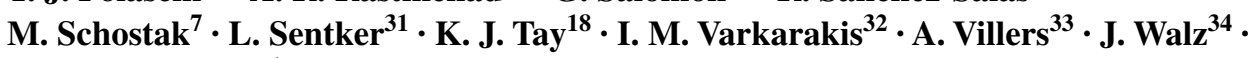 \\ J. J. De la Rosette ${ }^{1}$
}

Received: 31 December 2015 / Accepted: 2 February 2016 / Published online: 18 February 2016

(C) The Author(s) 2016. This article is published with open access at Springerlink.com

\begin{abstract}
Purpose To reach standardized terminology in focal therapy (FT) for prostate cancer (PCa).

Methods A four-stage modified Delphi consensus project was undertaken among a panel of international experts in the field of FT for PCa. Data on terminology in FT was collected from the panel by three rounds of online
\end{abstract}

Electronic supplementary material The online version of this article (doi:10.1007/s00345-016-1782-x) contains supplementary material, which is available to authorized users.

\section{A. W. Postema}

a.w.postema@amc.uva.nl

1 Departments of Urology, AMC University Hospital, Amsterdam, The Netherlands

2 Departments of Radiation Oncology, AMC University Hospital, Amsterdam, The Netherlands

3 USC Institute of Urology, Keck School of Medicine, University of Southern California, Los Angeles, CA, USA

4 Department of Urology, Kyoto Prefectural University of Medicine, Kyoto, Japan

5 Department of Urology, Angers University Hospital, Angers, France

6 Department of Urology, Institut Montsouris, Université Paris Descartes, Paris, France

7 Department of Urology, Magdeburg University Medical Center, Magdeburg, Germany

8 Department of Urology, Fuerth Hospital, Fuerth, Germany

9 Department of Radiation Oncology, Gustave Roussy Institute, Villejuif, France questionnaires. During a face-to-face meeting on June 21, 2015, attended by 38 experts, all data from the online rounds were reviewed and recommendations for definitions were formulated.

Results Consensus was attained on 23 of 27 topics; Targeted FT was defined as a lesion-based treatment strategy, treating all identified significant cancer foci; $F T$ was generically defined as an anatomy-based (zonal) treatment strategy. Treatment failure due to the ablative energy inadequately destroying treated tissue is defined as ablation

10 Department of Urology, Ospedale Civile Ramazzini, Carpi, Italy

11 Department of Surgery, Memorial Sloan-Kettering Cancer Center, New York, NY, USA

12 Department of Urology and Transplantation, Edouard Herriot Hospital, Lyon, France

13 Department of Urology, Instituto Valenciano de Oncología, Valencia, Spain

14 Department of Urology, University of Chicago, Chicago, IL, USA

15 Department of Urology, University of Leipzig, Leipzig, Germany

16 Joint Department of Medical Imaging, University Health Network, University of Toronto, Toronto, Canada

17 Departments of Radiology, Duke University Medical Center, Durham, NC, USA

18 Departments of Surgery, Duke University Medical Center, Durham, NC, USA

19 Urologische Praxis Dr. Henkel \& Dr. Kahmann, Berlin, Germany 
failure. In targeting failure the energy is not adequately applied to the tumor spatially and selection failure occurs when a patient was wrongfully selected for FT. No definition of biochemical recurrence can be recommended based on the current data. Important definitions for outcome measures are potency (minimum IIEF-5 score of 21), incontinence (new need for pads or leakage) and deterioration in urinary function (increase in IPSS $>5$ points). No agreement on the best quality of life tool was established, but UCLA-EPIC and EORTC-QLQ-30 were most commonly supported by the experts. A complete overview of statements is presented in the text.

Conclusion Focal therapy is an emerging field of PCa therapeutics. Standardization of definitions helps to create comparable research results and facilitate clear communication in clinical practice.

Keywords Focal therapy - Prostate cancer · Consensus · Definitions $\cdot$ Standardization $\cdot$ Outcome

20 Department of Urology, University of Heidelberg, Heidelberg, Germany

21 Glickman Urological and Kidney Institute, Cleveland Clinic, Cleveland, OH, USA

22 CamPARI Prostate Cancer Clinic, Cancer Directorate, Cambridge University Hospitals Trust, Cambridge, UK

23 Department of Urology, Theresien Krankenhaus Mannheim, Mannheim, Germany

24 Interdisciplinary Brachytherapy Unit, University of Lübeck, Lübeck, Germany

25 Division of Urology, Department of Experimental Medicine and Surgery, University of Rome Tor Vergata, Rome, Italy

26 Department of Urology, Free University Medical Centre, Amsterdam, The Netherlands

27 Department of Urology, University Hospital St Etienne, Saint-Étienne, France

28 Department of Urology, Porto Hospital Centre, Porto, Portugal

29 Department of Urology, Hofstra North Shore-Lij, Hofstra University, Hempstead, NY, USA

30 Martini-Clinic Prostate Cancer Center, University Hospital Hamburg-Eppendorf, Hamburg, Germany

31 Urologische Gemeinschaftspraxis, Sinsheim, Germany

32 2nd Department of Urology, Athens Medical University, University of Athens, Athens, Greece

33 Department of Urology, Lille University Medical Center, Lille, France

34 Department of Urology, Institut Paoli-Calmettes, Marseille, France

\section{Introduction}

Prostate cancer $(\mathrm{PCa})$ is traditionally treated with wholegland treatments such as radical prostatectomy, wholegland external beam radiotherapy and whole-gland brachytherapy [1]. Active surveillance allows selected patients with low-risk PCa to postpone or avoid radical treatment and the associated risk of toxicity [1]. Focal therapy (FT) is a fairly recent and rapidly developing field of PCa treatment where only a portion of the prostate gland is treated. Focal therapy intends to strike a balance between treating what must be treated while minimizing toxicity [2]. Different ablative energies employed for FT and under investigation include: cryosurgery, high-intensity focused ultrasound (HIFU), irreversible electroporation (IRE), laser ablation therapy, photodynamic therapy, and brachytherapy [3]. In the accumulating literature on FT, different terminology is used for different variants of tissue-sparing treatments, targeted lesions, and oncologic, functional and procedural outcomes. Standardization in definitions will aid in creating comparable research results in the literature and with clear communication in clinical settings. To achieve widely recognized standardized terminology in FT, we conducted an international multidisciplinary consensus project.

\section{Methods}

The Delphi method is a widely accepted method to achieve consensus among experts and is employed in economics, politics, military decision making and medicine [4]. The basis of the Delphi method is that a panel of experts is repeatedly presented a series of questions. Each successive round the question and answer possibilities are modified based on the responses to the previous round. The anonymous aggregated results and comments of the previous round are presented to the panel, allowing the participants to reassess their opinion. The intended outcome is a convergence of opinions with a minimized effect of peer-pressure and dominant individuals influencing group choices.

A systematic literature search of the English literature was conducted on "prostate cancer", "focal therapy" and the various FT modalities. The initial search yielded 190 results with subsequent automatic filtering, screening of titles, abstracts and full-texts resulting in the selection of 25 papers for data extraction. The search term and results are provided in Fig. 1. A group of 113 experts was invited to participate on the basis of the literature search and peer recommendation.

From the papers identified by the systematic search, various definitions of oncologic, functional and procedural outcomes were extracted and these data formed 
Fig. 1 Systematic search

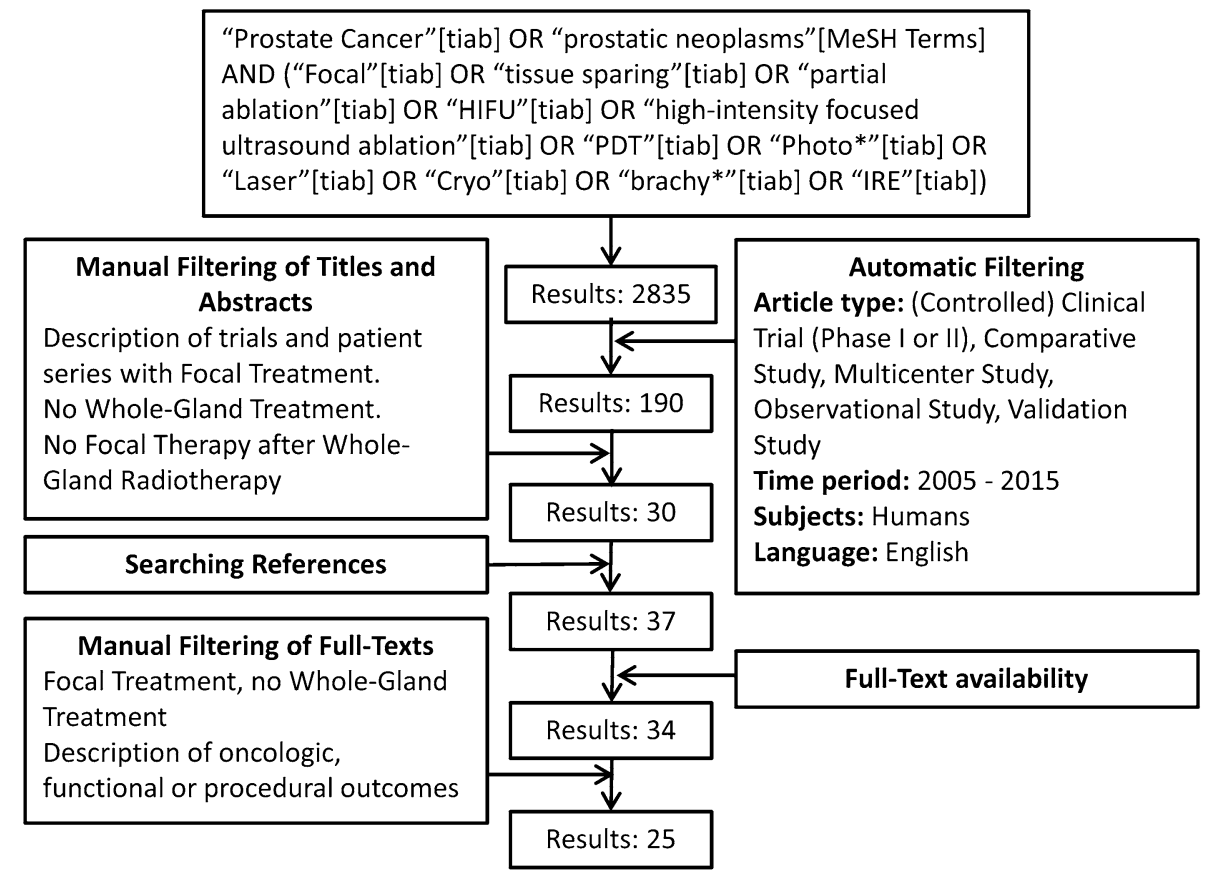

the basis of the questionnaires. The questionnaires constructed were presented to the participants in three successive rounds between May 15 and June 16, 2015 (using www.surveymonkey.com). The level of agreement necessary to achieve consensus was set at $80 \%$. During the 8th International Symposium on Focal Therapy and Imaging in Prostate \& Kidney Cancer (www.focaltherapy.org), a face-to-face meeting was held among 38 of the experts. All results from the online questionnaires were presented and discussed. Topics on which the online panel had achieved consensus were not overturned. Topics on which consensus had not been achieved were discussed in detail. Recommendations for definitions were formulated on all topics. An overview of the results of the online rounds and final recommendations for definitions is presented in Addendum 2.

\section{Results}

\section{Systematic search}

Twenty-five reports of clinical trials and trial protocols describing oncological, functional and procedural outcomes after a focal treatment, published after 2005, were identified by the systematic search. The modalities described were cryoablation [5-12], HIFU [6, 13-17], IRE [6, 13-21], laser ablation therapy [22-24], VTP [6, 25-28] and brachytherapy $[6,29]$.

\section{Treatment efficacy}

Commonly reported measures of treatment efficacy are prostate-specific antigen (PSA) dynamics after treatment $[5-17,20-22,25,26,28,29]$, the presence of suspicious areas on follow-up MRI [13, 14, 16, 18, 20-22, 25] and follow-up biopsy results. The planned follow-up biopsy scheme varies: in some protocols both the treated zone and the untreated zone are biopsied in every patient [5, 7-11, 15, 17, 19, 25-27, 29]. In other protocols, only the treated zone is biopsied [22,28], although some groups add biopsies from the untreated zone if new lesions are found on imaging [13, 14, 21]. Lee et al. [24] biopsied the ablated zone only at 3 months and performed a 12-core systematic biopsy and additional ablated zone biopsies at 1 year. Dickinson et al. [16] described treated zone biopsies at 1 year and template mapping biopsies at 3 years. Few authors define treatment success and failure, and the definitions vary: Ellis et al. [8] described an argument for only considering cancer found at follow-up in the treated zone or development of metastatic disease as treatment failure. Lindner et al. [27] referred to their four cases with residual tumor in the treated zone as treatment failures. Azzouzi et al. [25] defined a negative follow-up biopsy of the targeted zones as treatment success. In their protocol, Dickinson et al. [16] determined cancer control as the primary outcome using two definitions: (1) the combination of being free of any cancer in the treated zone and free of significant cancer in untreated zones at 3-year template 
biopsy or (2) free of significant disease in both treated and untreated zone at 3-year template biopsies. Onik et al. [11] proposed to define the combination of stable PSA and negative follow-up biopsy as a successful result. Durand et al. [7] defined treatment failure as positive follow-up biopsy in the treated lobe or biochemical failure according to the Phoenix criteria of PSA nadir $+2 \mathrm{ng} / \mathrm{mL}$ at 18 months.

\section{Trifecta}

Trifecta as a measure for urinary continence, potency and cancer control is defined by three papers, although two different definitions were used: pad-free, leak-free continence, erections sufficient for penetration and no high volume disease or Gleason $\geq 7$ disease on follow-up biopsies were used in two papers [13, 21]. Ahmeds' et al. [14] definition differed by substituting a radiologic outcome for the biopsy outcome of using no evidence of clinical disease on MRI at 12 months as a surrogate for cancer control.

\section{Significant cancer}

All papers that defined clinically significant disease at biopsy agreed that Gleason $\geq 7$ is significant at any cancer core length. The maximum length of Gleason 6 that is considered insignificant is $2 \mathrm{~mm}$ [14] or $3 \mathrm{~mm}$ [13, 16, 20, 21].

\section{Radiographic failure}

Several authors used imaging to determine whether residual tumor in the treated zone or new tumor outside the treated zone was present. Six papers reported the use of MRI [13, 14, 21, 22, 24, 29] and one additional paper [20] also defined criteria for suspicion of residual cancer: an early enhancing focus on the dynamic contrast sequence and residual restricted diffusion in the treatment area. Doppler ultrasound was used to target suspicious lesions post-treatment in one study [5]. One protocol described using both MRI and transrectal ultrasonography at 1, 3 and 5 years post-treatment without stating the purpose of these investigations [18].

\section{Biochemical status}

Baseline and post-treatment PSA dynamics are reported by most papers [5-22, 24-29]. The more specific "biochemical recurrence" is reported by few papers and different definitions were used: The original ASTRO definition of three consecutive rises above the nadir was used by five authors $[5,8,10,11,17]$. Durand et al. [7] used the Phoenix definition of PSA nadir $+2 \mathrm{ng} / \mathrm{mL}$. Lambert et al. [9] described two conditions, meeting either one was considered biochemical recurrence: PSA nadir $+2 \mathrm{ng} / \mathrm{mL}$ or a PSA nadir of less than $50 \%$ of the pre-treatment level. Nguyen et al. [29] advocated an alternative definition of biochemical recurrence where two conditions should both be met: PSA nadir $+2 \mathrm{ng} / \mathrm{mL}$ and a PSA velocity over $0.75 \mathrm{ng} / \mathrm{mL}$.

\section{Sexual function}

Sexual function is most commonly reported using the International Index of Erectile Function 5 item version (IIEF5), alternatively known as the Sexual Health Inventory for Men [6, 7, 15, 18, 19, 22, 24, 25, 27]. Durand et al. [7] defined an IIEF-5 score between 21 and 25 regardless of oral medication use as potent. The 15 item version (IIEF15) was used by four authors $[6-8,10]$. Two papers used the Brief Male Sexual Function Inventory BMSFI [5, 28, 30]. Six papers did not report the use of a validated questionnaire but did state the proportion of patients who were potent following FT [8-12, 20]. Four papers did not report sexual function [17, 23, 26, 29]. Different definitions for potency were in use besides the standardized questionnaires: the combination of achieving erections sufficient for vaginal penetration and being satisfied with sexual functioning regardless of the use of oral medication [10, 11], erections sufficient for vaginal penetration allowing phosphodiesterase- 5 inhibitors [8, 14], erections sufficient for vaginal penetration without stating whether medication is allowed $[9,13,20,21]$, and the ability to maintain an erection when stimulated with or without oral medication [5]. However, many papers that reported potency as an outcome did not state how it is defined $[6,12,15,16,18,19,22$, 24, 25, 27, 28]. Durand et al. [7] reported the number of patients who are sexually active before and after treatment without providing a definition.

\section{Urinary function}

The most commonly used measure of urinary function was the IPSS [6, 7, 13-19, 21, 22, 24, 25, 27, 28]. The UCLA-EPIC urinary domain was used by six papers $[13,14,16,17,19,21$, 24]. Continence was reported by several authors without providing an exact definition [6, 9, 12, 23, 24]. Commonly used definitions for incontinence were: the use of pads [7, 10, 11, $14,20]$, urinary leakage $[5,8]$ or combined: urinary leakage or pads $[13,14,21]$. One paper described the use of the International Continence Society questionnaire [15].

\section{Bowel function}

The UCLA-EPIC bowel domain was mentioned by two papers $[16,21]$. Other authors simply stated the absence of fistula, rectal discomfort, rectal bleeding, and change in frequency $[14,20,22]$. No definition for bowel toxicity in the context of FT was provided. 


\section{Quality of life}

Quality of life (QoL) was generally reported based on standardized questionnaires. The UCLA-EPIC [13, 14, $16,17,19,21,24,31]$ and FACIT (FACT-P and FACT-G) $[13,14,16,18,21,32,33]$ were most prevalent in the 25 papers identified by the literature search. Other questionnaires used were MAX-PC [16, 21, 34], EORTC-QLQ-30 [15, 35], PORPUS [27, 36] and EQ-5D [16, 21, 37]. No specific definitions or cut-off values for the questionnaires were provided.

\section{Complications}

For the timing and severity of complications, several definitions and systems were used. Perioperative or periprocedural complications were reported but not exactly defined by three papers $[19,22,27]$. Many authors used either the Clavien-Dindo score [6, 7] or the Common Terminology Criteria for scoring Adverse Events (CTCEA) scoring system to grade adverse events $[19,20,22,25]$. A CTCEA grade 3 and above and Clavien grade $3 \mathrm{a}$ and above were considered "severe". Moore et al. [28] described minor complications without providing a definition [28].

\section{Procedural outcomes}

Procedure time was reported by five papers and two did not provide a precise description [7, 13, 23]. Oto et al. [22] reported the total time the patient spends in the MR unit. Azzouzi et al. [25] reported the procedure time including anesthesia, targeting and ablation. Most papers stated how many days the urethral catheter was left in situ following FT [6-8, 12, 17-19, 22, 23, 27, 28]. Many authors provided the time the patient remained in the hospital after FT [6-8, 13, 14, 18-20, 22, 23, 27]. Dickinson et al. [16] implied that treatment in day-care per definition does not involve an overnight stay.

\section{Consensus project: response rates and participants}

One hundred thirteen experts were invited to participate. The response rate was $59 \%(67 / 113)$ for the first round, $53 \%(60 / 113)$ for the second and $65 \%(73 / 113)$ for the third round. Complete personal details were collected during the last online round and showed the following participant backgrounds: $75 \%$ were urologists, $11 \%$ radiologists, $4 \%$ radiotherapists, $4 \%$ researchers, $3 \%$ pathologists and $3 \%$ medical oncologists. The average number of patients treated with FT annually reported by the panelists was $10-50$ (47 \%), $7 \%$ of participants treated over 100 patients a year with FT. Cryosurgery, HIFU and brachytherapy were the most commonly used treatment modalities among the panel with 48,51 and $29 \%$ of the panelists reporting experience with these techniques. All consensus statements are summarized in Table 1. All registered participants to the online rounds and results of the online rounds discussed at the meeting are made available through Appendices 1 and 2 , respectively.

\section{General definitions}

The panel recommends defining "targeted focal therapy" as a lesion-based focal treatment of the target lesion(s) plus a safety margin. Ablating a quadrant, a lobe (hemiablation) or both lobes sub-totally would be defined as "focal therapy". Other variants of these anatomy-based focal therapy templates are subtotal ablation (any ablation where less than the whole gland is treated) and extended hemiablation (an ablation where one lobe is completely treated plus a margin of the other lobe, regardless of shape). The aim of targeted focal therapy should be the eradication of all identified significant tumors. There was agreement the index lesion is the single dominant lesion in terms of grade and size; grade being more important. Although there was agreement that there can be only 1 index lesion, the term "index lesion" itself may be of limited clinical use in the context of FT. When there are multiple significant lesions, it is more important to have an overview of all lesions that require treatment rather than a single defined index lesion.

There was no consensus on the definition of salvage focal therapy during the online rounds. After discussion at the meeting, the panel supported the following statement: Salvage focal therapy refers to a situation where focal treatment is applied to the prostate after previous whole-gland therapy, or in the same region of the prostate as a previous FT. The prostate gland has to be in place.

\section{Success and failure in focal therapy}

In defining success and failure of FT, two levels were observed by the panel during the meeting: Failure of the focal treatment as a whole and several reasons for a focal treatment to fail ("ablation failure", "targeting failure" and "selection failure"). Ablation failure is a failure of the technique to destroy the tissue within the intended treated zone, evidenced by tumor detected within the treated zone. Ablation failure must be confirmed histologically. Any cancer left in the treated zone only is termed "residual disease". Radiographic suspicion of ablation failure is imaging suspect for tumor presence within the treated zone. The panelists unanimously agreed that multiparametric $(\mathrm{mp})$ MRI is a suitable imaging tool for monitoring this, followed by $20 \%$ for CEUS and $16 \%$ for PET. Targeting failure occurs when the ablative energy is not correctly applied to the tumor spatially and for selection failure, FT was 
Table 1 Definitions and consensus statements

Definition Consensus statement regarding definition

General definitions

Focal therapy (FT)

\section{Targeted FT}

The aim of (targeted) FT for PCa

Subtotal ablation

Extended-hemiablation

Index lesion

Salvage FT

Success and failure in focal therapy

Ablation failure

Radiographic suspicion of ablation failure

Residual disease

Selection failure

Biochemical progression after targeted FT

Pathological progression

Baseline and outcome functional measures Functional success of FT

Erectile function

Sexually active

Urinary incontinence

Significant deterioration of urinary function Quality of life

Bowel toxicity/GI side effects

Intraoperative complications

Short-term side effects

Serious side effects

Procedural outcomes

Procedure time
An anatomy-based (zonal) treatment strategy (e.g. targeting a quadrant, a lobe or both lobes sub-totally)

A lesion-based focal treatment strategy targeting the identified tumors plus a safety margin

Eradication of all significant cancer(s)

Any ablation where less than the whole gland is treated

An ablation where one lobe is completely treated plus a margin of the other lobe regardless of shape

The single dominant lesion in terms of grade and size where grade is more important. There can be only 1 index lesion. The term index lesion itself may be of limited use in the context of FT. It is more important to have an overview of all significant lesions that warrant treatment rather than a single defined index lesion

Salvage FT refers to the situation where FT is applied to the prostate after whole-gland therapy, or in the same region of the prostate as previous FT. The prostate gland has to be in place

Ablation failure is a failure of the technique to destroy the tissue in the treated zone, evidenced by tumor found within the treated zone. Ablation failure is just one of the causes that can lead to failure of FT as a whole. Other types of failure include targeting failure and selection failure. Must be confirmed by targeted biopsy

A suspicion on imaging of tumor presence within the treated zone. mpMRI a suitable imaging modality to determine ablation failure

Cancer remaining in the target zone after FT

FT was inappropriately indicated, evidenced by short-term post-treatment identification of metastatic or locally advanced disease. There is no agreement on whether significant PCa in shortterm biopsies taken inside or outside the treatment zone and the need for whole-gland treatment during follow-up constitute selection failure

PSA is the best marker to monitor the disease after targeted FT. However, there is currently no data on how to use PSA, i.e. there is no data to support any of the definitions for biochemical recurrence in the context of (targeted) FT

An increase in Gleason score or tumor volume evidenced by a larger number of positive biopsies or larger per-core tumor involvement

The maintenance of voiding pattern, erectile function and quality of life assessed after 12 months

A qualitative definition of impotency exists: the persistent inability to attain and maintain an erection sufficient for satisfactory sexual performance. For reporting research the panel recommends defining significant erectile dysfunction using the IIEF-5 score $<21$, determined at 1 year

Patient-reported regular sexual activity

The need to use pads or patient-reported leakage. More comprehensive data could be gathered by requesting patients to complete a micturition diary including the parameters: number of pads, leakage and urge

An increase in IPSS $>5$ points

A quality-of-life questionnaire should be administered and both the UCLA-EPIC and the EORTCQLQ-c-30 tools can be used although neither one is validated for the specific context of focal therapy

The occurrence of: a change in stool frequency, fistula formation, soiling and/or blood in the stool after FT should constitute bowel toxicity/GI side effects. There is no consensus on whether mucus in the stool should also be included. The use of one of the existing grading systems for bowel toxicity is recommended

Complications that cause damage to the patients' health or require intervention to prevent damage Side effects within 90 days of the procedure

Clavien-Dindo-scale with 3 or greater as "serious" side effects

From the completion of anesthetic induction until the treating physician is finished 
Table 1 continued

\begin{tabular}{ll}
\hline Definition & Consensus statement regarding definition \\
\hline Hospital stay & The time from admittance until discharge \\
FT in day-care & Admittance, treatment and discharge on the same day \\
Catheterization time & $\begin{array}{l}\text { The time from inserting the catheter until its removal, including time spent on the OR and the } \\
\text { recovery-unit }\end{array}$ \\
\hline
\end{tabular}

All statements were accepted with $>80 \%$ consensus unless stated other otherwise

inappropriately indicated to the patient. The panel agreed that selection failure was definitely evidenced by shortterm post-treatment identification of metastatic or locally advanced disease. However, during the meeting there was no agreement on how selection failure could be inferred from post-treatment biopsy results. There was agreement that PSA is the best marker to monitor disease activity after (targeted) FT, even though there is currently no data on how to use PSA after focal treatments where (malignant) prostate tissue is left in place. Therefore, there is no data to support any of the definitions for biochemical recurrence commonly used after whole-gland treatment in the context of (targeted) FT. In defining pathological progression the panel agrees that both an increase in Gleason score and tumor volume evidenced by a growing number of positive biopsies or larger per-core tumor involvement should be taken into consideration.

\section{Baseline and outcome functional measures}

The panel recommended defining functional success of focal therapy as the maintenance of voiding pattern, erectile function and QoL assessed after 12 months. In defining potency, most of the online panel agreed a minimum IIEF score should be used. During the face-to-face meeting, it was stressed that a widely endorsed qualitative definition of impotency already exists: "the persistent inability to attain and maintain an erection sufficient for satisfactory sexual performance". However, a quantitative definition is useful in reporting and this quantitative definition should be based on the IIEF-5. The panel recommended defining significant erectile function following FT using the IIEF-5 score with a cut-off value of 21 , determined at 1 year. The definition of sexually active should be based on patient-reported sexual activity.

Urinary incontinence is defined as the need to use pads or patient-reported leakage. During the face-to-face meeting, it was suggested that more comprehensive data should be gathered by requesting patients to fill in a voiding diary including: number of pads, leakage and urgency. The recommended definition of significant deterioration of urinary function is an IPSS increase $>5$ points.
In defining the QoL status of patients before and after FT different tools can be used. None of the tools were supported by more than $80 \%$ of the panelists. The EORTCQLQ-C-30 was supported by $75 \%$ of the panel and the UCLA-EPIC by $70 \%$. During the face-to-face meeting, there was agreement that a QoL questionnaire should be although neither one is validated in the specific context of FT.

In defining "bowel toxicity" following FT, the panel agreed that a change in stool frequency, fistula formation, or the occurrence or increase of soiling and blood in the stool after FT constitutes bowel toxicity. There is no consensus on whether the occurrence or increased production of mucus in the stool should be included. During the face-to-face meeting, a recommendation was formulated to use either one (CTCEA or RTOG/EORTC) of the existing grading systems for bowel toxicity. There was agreement that it may not be necessary to present a full questionnaire to all patients but only to those who have indicated that they have gastro-intestinal side effects.

The recommended definition of short-term side effects is: side effects that become apparent within 90 days after the procedure. The panel recommended including only complications to the definition of intraoperative complications that cause damage to the patients' health or require a subsequent intervention. Therefore, technical difficulties with the equipment and targeting difficulties due to anatomy are not necessarily intraoperative complications. The panel agreed to grade side effects using the ClavienDindo-scale regarding a grade 3 and higher as "serious side effects".

\section{Procedural outcomes}

The panel recommended defining procedural time as the time period starting after the anesthetic induction is completed and the treating physician can start until the treating physician has finished the treatment. Hospital stay should be defined as the time from admission until discharge. The definition of FT in day-care should be: admittance, treatment and discharge on the same calendar day. The definition of catheterization time should be the time from 
inserting the catheter until its removal, including time spent on the OR and the recovery-unit.

\section{Discussion}

The results of this Delphi consensus project will aid researchers in reporting FT outcomes in a standardized fashion, thereby allowing comparison and also facilitating clear communication among patients, clinicians and researchers. As standardized definitions can only succeed if they are widely endorsed by the experts in the field, a large-group consensus project is the ideal way to achieve this. Consensus projects can be a valuable tool, especially in fields such as FT where the clinical evidence is still building. It is therefore not surprising that several consensus projects on various aspects of FT have been undertaken in the past years. The topics handled in our consensus project partly overlap with the topics of other consensus meetings. There appears to have been a shift in the perceived aim of FT. The 2009 consensus project described by De la Rosette et al. [38] stipulated that the aim of FT is to eradicate all known prostate cancer while preserving uninvolved tissue, sparing genitourinary function. The 2010 consensus project described by Ahmed et al. [39] stated the aim of FT is to treat cancer and leave benign prostate and surrounding normal structures. They do not note the possibility to also leave insignificant disease untreated. Five years later, we formulated the aim of FT to be the eradication of all identifiable significant tumor(s). The consensus project described by Donaldson et al. [40] stated that FT should be the treatment of the dominant lesion or index lesion. They add that quadrant ablation could be a FT strategy but with lower level of consensus than lesion ablation only. This may imply their panel considered FT as a lesion-based technique, similar to our definition of targeted FT. Consistent with the concerns regarding secondary (non-index) lesions harboring clinically significant disease with metastatic potential formulated by Reis et al. [41], the participants of our consensus project underlined the necessity to find and treat all clinically significant cancer foci, regardless of the precise definition of the index lesion.

The consensus projects described by Muller et al. [42] and De la Rosette et al. [38] provided definitions for oncologic success and oncologic efficacy, respectively, that are similar to our definition of ablation success: negative biopsies in the treated area. The definitions around the concepts of success and failure provided by the consensus project described by Van den Bos et al. [43] differed slightly from ours; they recommended defining in-field failure as: (1) higher Gleason grade disease in the treated zone, (2) persistent cancer of similar or lower grade after repeat FT of the same area and (3) the need for additional treatment besides
FT because of objective findings elsewhere in the gland. While the first two conditions are concordant with our definition of ablation failure, the latter does not. Finding tumor for which FT is not suitable during short-term follow-up elsewhere in the gland would constitute selection failure by our definition. Their definition of selection failure appears not to be related to adequate patient selection for FT but to the selection of tumor foci for treatment.

The project described by Muller et al. [42] described a definition of functional success comparable to ours: the absence of functional change in erectile function and ejaculatory function at 24 months, QoL at 24 months and urinary function at 12 months. The main difference is that present consensus does not include ejaculatory function and only uses the 12-month time point for all three domains of functional outcomes.

The consensus projects by Muller et al. [42] and Van den Bos et al. [43] provided recommendations for follow-up after FT. Similar to our results, they recommended to monitor PSA, although they also found no standardized definition of biochemical recurrence could be recommended. Other follow-up parameters include urinary function, sexual function and quality of life using standardized tools, including IPSS and IIEF questionnaires. In the reviewed literature, FACIT (FACT-P) and (FACT-G) and UCLAEPIC questionnaires were the most prevalent tools for the assessment of QoL following FT. Muller et al. [42] could not achieve consensus on which QoL tool to be used, but the FACT-P was widely supported. In the project described by Van den Bos et al. [43], the UCLA-EPIC was recommended, a least for pre-treatment assessment of QoL. De la Rosette et al. [38] also recommended to use the FACT$\mathrm{P}$ and EORTC questionnaire among several other options. In our project the EORTC-QLQ-c30 was widely endorsed besides the UCLA-EPIC, while the FACIT questionnaire was considered useful by only $50 \%$ of our online panel. MpMRI is universally recommended as a tool for followup after FT, which is consistent with our panel agreeing unanimously that mpMRI is a suitable tool to define radiographic suspicion of ablation failure.

Several considerations regarding our consensus project are: as the results are based on expert opinion they amount to an Oxford Centre of Evidenced Based Medicine level 5 of evidence [44]. The group of experts we invited may not be representative of the larger medical community as their involvement in FT makes them more likely to be enthusiasts. Furthermore, the response rates to our online questionnaires were between 59 and $65 \%$, although this is likely caused by our attempt to invite a large and broad group of experts, further bias in our results might be caused by "non-believers" not participating. Although a total of 38 experts joined the face-to-face meeting, which took place at a FT meeting, their opinions may be more prominently 
represented in the final recommendations than the opinions of the participants of the online rounds only. The repetitive formulation and reformulation of questions and answer possibilities by the project leaders may also be a source of bias and can be seen as an inevitable limitation of the Delphi method.

Several important topics remain undefined because of consensus: (1) there was insufficient data (2) unresolved diverging opinions within the panel or (3) the topics were un-addressed by the current project. Examples of important unresolved matters are: "What constitutes a clinically significant tumor focus?", "How should PSA dynamics be interpreted after FT?", "How can QoL best be assessed after FT" and "What are adequate safety margins to be observed for the different treatment modalities?". These topics can be addressed in future consensus projects. As the data on FT accumulates, it should become possible to formulate stronger recommendations and data-based guidelines on how best to research, apply and report focal treatments.

\section{Conclusion}

Focal therapy is a rapidly evolving field of prostate cancer treatments that intends to prevent or delay wholegland treatment associated morbidity without compromising oncologic safety for a large group of patients. For the development and implementation of these treatments, it is important to have standardized reporting criteria. The current consensus project provides recommendations for standardized definitions endorsed by a wide group of experts in the field.

Acknowledgments This project was made possible by financial support of the Dutch Cancer Society and the Cure for Cancer foundation.

Author contributions A.W. Postema, T.M. de Reijke, O. Ukimura, and J.J. De la Rosette were involved in project development, data collection, data analysis, and manuscript writing/editing; W. Van den Bos was involved in project development and manuscript writing/editing; A.R. Azzouzi, E. Barret, D. Baumunk, A. Blana, A. Bossi, M. Brausi, J.A. Coleman, S. Crouzet, J. Dominguez-Escrig, S. Eggener, R. Ganzer, S. Ghai, I.S. Gill, R.T. Gupta, T.O. Henkel, M. Hohenfellner, J.S. Jones, F. Kahmann, C. Kastner, K.U. Köhrmann, G. Kovacs, R. Miano, R.J. van Moorselaar, N. Mottet, L. Osorio, B.R. Pieters, T.J. Polascik, A.R. Rastinehad, G. Salomon, R. Sanchez-Salas, M. Schostak, L. Sentker, K.J. Tay, I.M. Varkarakis, A. Villers and J. Walz were involved in data collection, data analysis and manuscript editing.

\section{Compliance with ethical standards}

This is a report of a Delphi consensus project among experts only. No human or animal subjects were involved; therefore, no informed consent was collected.
Conflict of interest O.U reports being a consultant for Sonacare Medical. A.A. reports consulting for Steba Biotech. A.Bl., M.S., R.S. and S.C. report being consultants for EDAP TMS. S.E. reports being a consultant for NxThera and Profound Medical. J.J. reports being consultant for Endocare. C.K. reports being a consultant for Elekta and organizing educational internships sponsored by Elekta, Siemens, Philips, Medicom and Hitachi. T.P. reports performing a clinical trial with Angiodynamics and being a consultant for Healthtronics. The other authors report no conflicts of interest.

Open Access This article is distributed under the terms of the Creative Commons Attribution 4.0 International License (http://creativecommons.org/licenses/by/4.0/), which permits unrestricted use, distribution, and reproduction in any medium, provided you give appropriate credit to the original author(s) and the source, provide a link to the Creative Commons license, and indicate if changes were made.

\section{References}

1. Mottet N, Bellmunt J, Patient EB, Associate RCNVDBG, Culine S, Joniau S et al (2015) Guidelines on prostate cancer. Eur Urol. http://uroweb.org/guideline/prostate-cancer/

2. Valerio M, Ahmed HU, Emberton M, Lawrentschuk N, Lazzeri M, Montironi R et al (2013) The Role of focal therapy in the management of localised prostate cancer: a systematic review. Eur Urol 44:1-20

3. van den Bos W, Muller BG, Ehdaie B, Scardino P, de la Rosette JJMCH (2014) What is still needed to make focal therapy an accepted segment of standard therapy? Curr Opin Urol 24(3):247-255

4. Hsu C, Sandford B (2007) The Delphi technique: making sense of consensus. Practical Assess Res Eval 12(10):1-8

5. Bahn DK, Silverman P, Lee F, Badalament R, Bahn ED, Rewcastle JC (2006) Focal prostate cryoablation: initial results show cancer control and potency preservation. J Endourol 20(9):688-692

6. Barret E, Ahallal Y, Sanchez-Salas R, Galiano M, Cosset JM, Validire P et al (2013) Morbidity of focal therapy in the treatment of localized prostate cancer. Eur Urol 63:618-622

7. Durand M, Barret E, Galiano M, Rozet F, Sanchez-Salas R, Ahallal Y et al (2014) Focal cryoablation: a treatment option for unilateral low-risk prostate cancer. BJU Int 113:56-64

8. Ellis DS, Manny TB, Rewcastle JC (2007) Focal cryosurgery followed by penile rehabilitation as primary treatment for localized prostate cancer: initial results. Urology 70:9-15

9. Lambert EH, Bolte K, Masson P, Katz AE (2007) Focal cryosurgery: encouraging health outcomes for unifocal prostate cancer. Urology 69:1117-1120

10. Onik G (2007) Rationale for a "male lumpectomy", a prostate cancer targeted approach using cryoablation: results in 21 patients with at least 2 years of follow-up. Cardiovasc Interv Radiol 2008(31):98-106

11. Onik G, Vaughan D, Lotenfoe R, Dineen M, Brady J (2008) The "male lumpectomy": focal therapy for prostate cancer using cryoablation results in 48 patients with at least 2-year follow-up. Urol Oncol Semin Orig Investig 26:500-505

12. Rosenberg GS, Basralian KG (2010) Active hydrodissection might optimize cryosurgical ablation of the prostate. Urology 76(4):988-991. doi:10.1016/j.urology.2009.12.081

13. Ahmed HU, Freeman A, Kirkham A, Sahu M, Scott R, Allen C et al (2011) Focal therapy for localized prostate cancer: a phase I/II trial. J Urol 185(4):1246-1254. doi:10.1016/j. juro.2010.11.079 
14. Ahmed HU, Hindley RG, Dickinson L, Freeman A, Kirkham AP, Sahu M et al (2012) Focal therapy for localised unifocal and multifocal prostate cancer: a prospective development study. Lancet Oncol 13(6):622-632. doi:10.1016/S1470-2045(12)70121-3

15. Crouzet S, Rouviere O, Martin X, Gelet A (2014) High-intensity focused ultrasound as focal therapy of prostate cancer. Curr Opin Urol 24(3):225-230

16. Dickinson L, Ahmed HU, Kirkham AP, Allen C, Freeman A, Barber J et al (2013) A multi-centre prospective development study evaluating focal therapy using high intensity focused ultrasound for localised prostate cancer: the INDEX study. Contemp Clin Trials 36(1):68-80. doi:10.1016/j.cct.2013.06.005

17. Muto S, Yoshii T, Saito K, Kamiyama Y, Ide H, Horie S (2008) Focal therapy with high-intensity-focused ultrasound in the treatment of localized prostate cancer. Jpn J Clin Oncol 38(3):192-199

18. van den Bos W, Muller BG, de la Rosette JJMCH (2013) A randomized controlled trial on focal therapy for localized prostate carcinoma: hemiablation versus complete ablation with irreversible electroporation. J Endourol 27(3):261-4. http://online. liebertpub.com/doi/abs/10.1089/end.2013.1568

19. van den Bos W, de Bruin DM, Muller BG, Varkarakis IM, Karagiannis AA, Zondervan PJ et al (2014) The safety and efficacy of irreversible electroporation for the ablation of prostate cancer: a multicentre prospective human in vivo pilot study protocol. BMJ Open 4(10):e006382-e006382. http://bmjopen.bmj.com/cgi/ doi/10.1136/bmjopen-2014-006382

20. Valerio M, Stricker PD, Ahmed HU, Dickinson L, Ponsky L, Shnier R et al (2014) Initial assessment of safety and clinical feasibility of irreversible electroporation in the focal treatment of prostate cancer. Prostate Cancer Prostatic Dis 17(4):343-347

21. Valerio M, Dickinson L, Ali A, Ramachandran N, Donaldson I, Freeman A et al (2014) A prospective development study investigating focal irreversible electroporation in men with localised prostate cancer: nanoknife electroporation ablation trial (NEAT). Contemp Clin Trials 39:57-65

22. Oto A, Sethi I, Karczmar G, McNichols R, Ivancevic MK, Stadler WM et al (2013) MR imaging—guided focal laser ablation for prostate cancer : phase I trial. Radiology 267(3):932-940

23. Napoli A, Anzidei M, De Nunzio C, Cartocci G, Panebianco V, De Dominicis C et al (2013) Real-time magnetic resonanceguided high-intensity focused ultrasound focal therapy for localised prostate cancer: preliminary experience. Eur Urol 63(2):395-398

24. Lee T, Mendhiratta N, Sperling D, Lepor H. (2014) Focal laser ablation for localized prostate cancer: principles, clinical trials, and our initial experience. Rev Urol 16(2):55-66

25. Azzouzi AR, Barret E, Moore CM, Villers A, Allen C, Scherz A et al (2013) TOOKAD ${ }^{\circledR}$ Soluble vascular-targeted photodynamic (VTP) therapy: determination of optimal treatment conditions and assessment of effects in patients with localised prostate cancer. BJU Int 112(6):766-774

26. Eymerit-Morin $\mathrm{C}$, Zidane M, Lebdai S, Triau S, Azzouzi AR, Rousselet M-C (2013) Histopathology of prostate tissue after vascular-targeted photodynamic therapy for localized prostate cancer. Virchows Arch 463(4):547-552

27. Lindner U, Weersink RA, Haider MA, Gertner MR, Davidson SRH, Atri M et al (2009) Image guided photothermal focal therapy for localized prostate cancer: phase I trial. J Urol 182(4):1371-1377. doi:10.1016/j.juro.2009.06.035

28. Moore CM, Nathan TR, Lees WR, Mosse CA, Freeman A, Emberton $M$ et al (2006) Photodynamic therapy using meso tetra hydroxy phenyl chlorin (mTHPC) in early prostate cancer. Lasers Surg Med 38(5):356-363
29. Nguyen PL, Chen M, Zhang Y, Tempany CM, Cormack RA, Beard CJ et al (2013) Implications for focal. Therapy 188(4):1151-1156

30. Rosen RC (1996) Brief male sexual function inventory for urology. Urology 47(5):782-783

31. Litwin MS, Hays RD, Fink A, Ganz PA, Leake B, Brook RH (1998) Prostate cancer index and validity quality. Med Care 36(7):1002-1012

32. Esper P, Mo F, Chodak G, Sinner M, Cella D, Pienta KJ (1997) Measuring quality of life in men with prostate cancer using the functional assessment of cancer therapy-prostate instrument. Urology 50(6):920-928

33. Overcash J, Extermann M, Parr J, Perry J, Balducci L (2001) Validity and reliability of the FACT-G scale for use in the older person with cancer. Am J Clin Oncol 24(6):591-596

34. Roth A, Nelson CJ, Rosenfeld B, Warshowski A, O'Shea N, Scher $\mathrm{H}$ et al (2006) Assessing anxiety in men with prostate cancer: further data on the reliability and validity of the Memorial Anxiety Scale for Prostate Cancer (MAX-PC). Psychosomatics 47(4):340-347

35. Aaronson NK, Ahmedzai S, Bergman B, Bullinger M, Cull A, Duez NJ et al (1993) The European Organization for Research and Treatment of Cancer QLQ-C30: a quality-of-life instrument for use in international clinical trials in oncology. J Natl Cancer Inst 85(5):365-376

36. Ritvo P, Irvine J, Naglie G, Tomlinson G, Bezjak A, Matthew A et al (2005) Reliability and validity of the PORPUS, a combined psychometric and utility-based quality-of-life instrument for prostate cancer. J Clin Epidemiol 58(5):466-474

37. What is EQ-5D [Internet]. [cited 2015 Jul 31]. http://www.euroqol.org/

38. de la Rosette J, Ahmed H, Barentsz J, Johansen TB, Brausi M, Emberton $\mathrm{M}$ et al (2010) Focal therapy in prostate cancer-report from a consensus panel. J Endourol 24(5):775-780

39. Ahmed HU, Akin O, Coleman JA, Crane S, Emberton M, Goldenberg L et al (2012) Transatlantic Consensus Group on active surveillance and focal therapy for prostate cancer. BJU Int 109(11):1636-1647

40. Donaldson IA, Alonzi R, Barratt D, Barret E, Berge V, Bott $S$ et al (2015) Focal therapy: patients, interventions, and outcomes - a report from a consensus meeting. Eur Urol 67(4): 771-7. http://linkinghub.elsevier.com/retrieve/pii/ S0302283814009555

41. Reis LO, Billis A, Zequi SC, Tobias-Machado M, Viana P, Cerqueira $M$ et al (2014) Supporting prostate cancer focal therapy: a multidisciplinary International Consensus of Experts ("ICE"). Aging Male 17(2):66-71. http://www.scopus.com/inward/ record.url?eid=2-s2.0-84901269492\&partnerID $=40 \& \operatorname{md5}=0 \mathrm{~d} 0$ d244d38d60d2078f918ece972ff25

42. Muller BG, van den Bos W, Brausi M, Fütterer JJ, Ghai S, Pinto PA et al (2015) Follow-up modalities in focal therapy for prostate cancer: results from a Delphi consensus project. World J Urol. http://link.springer.com/10.1007/s00345-014-1475-2

43. Van Den Bos W, Muller BG, Ahmed H, Bangma CH, Barret E, Crouzet $S$ et al (2014) Focal therapy in prostate cancer: international multidisciplinary consensus on trial design. Eur Urol 65(6):1078-1083

44. Oxford CEBM Levels of evidence [Internet]. http://www.cebm. net/ocebm-levels-of-evidence/ 